\title{
Coffee protection against the development of hepatocellular carcinoma: review article
}

\author{
Kellyane S. D. Carvalho, Helma P. Cotrim \\ Programa de Pós-Graduação em Medicina e Saúde, Faculdade de Medicina - Universidade Federal da Bahia, Bahia 40110-060, Brazil. \\ Correspondence to: Dr. Helma P. Cotrim, Programa de Pós-Graduação em Medicina e Saúde - PPGMS, Complexo Universitário Professor \\ Edgard Santos, Rua Augusto Viana, 5o. andar; Canela, Salvador, Bahia 40110-060, Brazil. E-mail: helmacotrim@gmail.com
}

How to cite this article: Carvalho KSD, Cotrim HP. Coffee protection against the development of hepatocellular carcinoma: review article. Hepatoma Res 2020;6:6. http://dx.doi.org/10.20517/2394-5079.2019.027

Received: 8 Nov 2019 First Decision: 13 Dec 2019 Revised: 20 Jan 2020 Accepted: 6 Feb 2020 Published: 21 Feb 2020

Science Editor: Dalbir Sandhu Copy Editor: Jing-Wen Zhang Production Editor: Tian Zhang

\begin{abstract}
Coffee, a popular drink around the world, is composed of a complex mix of biologically active molecules, including caffeine, chlorogenic acid, and diterpenes. These compounds have antioxidant, anti-inflammatory, antifibrotic, and anticarcinogenic properties, which may explain observational data showing that coffee drinkers have lower rates of chronic liver disease, including cirrhosis and hepatocellular carcinoma (HCC). Recent studies have also shown that coffee consumption may also increase patient survival before and after liver transplantation. The mechanism by which coffee consumption protects against HCC is not clear; however, its relevant role has been demonstrated. This literature review article focuses on the role of coffee consumption in protecting against the development of HCC. Methodology: Scientific articles indexed through PubMed, including Medline, Scielo, and Lilacs, published in English were used as search methods. The terms used in English were: "hepatocellular carcinoma" or "Liver cancer" or "HCC" and "coffee". According to the study design or review article, cross-sectional, longitudinal, or descriptive investigations were included, showing site and year of publication until 2019.
\end{abstract}

Keywords: Hepatocellular carcinoma, coffee consumption protection, liver cancer

\section{INTRODUCTION}

Hepatocellular carcinoma (HCC) is the main and most frequent malignant liver tumor today. Its prevalence has been increasing steadily around the world in recent decades ${ }^{[1]}$, and its development is associated with chronic liver diseases caused by hepatitis B and C viruses, alcoholic cirrhosis, non-alcoholic steatohepatitis, and metabolic diseases such as primary hemochromatosis ${ }^{[2,3]}$. 
Coffee is one of the most consumed beverages in the world. Coffee consists of several components, such as caffeine, triacylglycerol, tocopherols, chlorogenic acid, cafestol, and kahweol, with relevant antioxidant and anti-inflammatory properties. Because of their biological properties, these substances can have beneficial effects on metabolism ${ }^{[4]}$.

For many years, coffee intake has been related to adverse effects, mainly cardiovascular, but recent studies show that daily coffee consumption, in addition to being harmful, can be beneficial and protective, especially against the development of chronic liver diseases such as HCC. This protection seems to be associated with the habit and frequency of coffee consumption ${ }^{[5]}$.

As most studies on this subject have been observational, the mechanisms of coffee protection against various diseases remain uncertain. However, some hypotheses have been suggested: polyphenols, antioxidants found in coffee, may protect cells against oxidative stress and damage to DNA molecules ${ }^{[6]}$. The protection conferred by coffee is believed to occur through antioxidant components, which would influence the lower liver enzymatic activity. Gamma-glutamyl transpeptidase (GGT) and alanine aminotransferase (ALT) in these studies showed lower serum levels in coffee drinkers versus nondrinkers ${ }^{[7]}$.

The importance of hepatocarcinogenic pathways for the development of HCC has also been discussed, and molecular mechanisms that generate liver aggression and metabolic changes in hepatocytes related mainly to oxidative stress have been observed. The accumulation of several oxidative metabolites directly attacks DNA molecules or makes the general functioning of the cell unfeasible, generating apoptosis. Thus, it is observed that the protective mechanisms of substances present in coffee antagonize the hepatic carcinogenic pathways, which theoretically supports the findings of coffee consumption as a protector of the liver.

Even with the accumulation of evidence in favor of coffee consumption, its dietary prescription remains controversial. It is estimated that $36 \%$ of health professionals still believe that coffee consumption increases the risk of cancer. Thus, research that reinforces the benefits of coffee is still needed, especially when the outcomes are more impactful and chronic, such as the incidence of hepatocellular carcinoma ${ }^{[8]}$.

This literature review aims to evaluate the role of coffee consumption in protecting against the development of HCC.

\section{METHODOLOGY}

Scientific articles indexed through PubMed, including Medline, SciELO, and Lilacs, published in English were used as search methods.

The terms used in English were: "hepatocellular carcinoma" or "liver cancer" or "HCC and "coffee". According to the study design or review article, cross-sectional, longitudinal, or descriptive investigations were included, showing site and year of publication until 2019.

This literature review included articles that evaluated the role of coffee consumption in protecting against the development of HCC and included the following topics: HCC pathogenesis; caffeine and HCC; amount of coffee consumed and protective effect on hepatocarcinogenesis; impact of coffee consumption on HCC mortality; and coffee consumption and recurrence of HCC after liver transplantation.

\section{HCC PATHOGENESIS}

The pathogenesis of HCC is multifactorial, which initially includes an inflammatory process mediated by Kupffer cells in the liver or macrophages, which stimulate the production of proinflammatory 
cytokines [interleukin 6 (IL-6) and tumor necrosis factor (TNF- $\alpha$ )] and immunosuppressive cytokines ${ }^{[9,10]}$ (IL-10). The accumulation of these immunostimulating agents around the liver inflammation focus induces mitochondrial imbalance in hepatocytes (increased oxygen uptake and increased production of superoxide anions, hydroxyl radicals, and oxide), leading to the production of high levels of reactive oxygen species (ROS) and a consequent increase in oxidative stress ${ }^{[11,12]}$.

In addition, hepatic mitochondria are also essential in hepatocyte survival as the mediator of apoptosis and necrosis, controlling the balance between cell survival and death by regulating membrane permeability, and activating the intrinsic pathway of apoptosis (cytochrome $\mathrm{C}$ protein release, apoptosis formation, and caspase activation ${ }^{[9]}$ ). In addition, increased ROS production causes oxidative damage to mitochondrial proteins (impairing ATP synthesis) and alters the induction of pore production from mitochondrial transition permeability. These changes make the inner membrane permeable to small molecules that can cause ischemia or reperfusion injury and DNA damage by activating the intrinsic apoptotic mechanism ${ }^{[13]}$.

These mechanisms can induce recurrent cycles of cell damage, repair, and regeneration in hepatocytes, leading to the formation of dysplastic nodular lesions, which are precursor lesions of HCC. Associated with these changes are genetic and epigenetic changes that are directly related to tumor progression and HCC ${ }^{[14]}$.

\section{CAFFEINE AND HCC}

Caffeine, one of the components of coffee, appears to play a central role in protecting against the development of chronic liver disease and HCC. It reduces HCC cell proliferation, and it has been observed that protection against HCC is lower for decaffeinated coffee ${ }^{[15]}$.

Other substances, such as cafestol and kahweol, tirpenoid molecules present in coffee beans, increase the activity of liver enzymes, which may improve metabolism and excretion of carcinogens ${ }^{[16,17]}$. However, cafestol and kahweol are only present in minimal quantities in filtered coffee, and these coffee varieties are popular in countries, such as Japan and Finland, where studies have shown inverse associations with $\operatorname{HCC}^{[18,19]}$.

Specific coffee protection mechanisms may include inhibition of viral hepatitis activity and prevention of diabetes mellitus type 2. In addition, coffee has a number of health benefits, including a lower incidence of neurological diseases, various cancers, and reduced mortality from any cause $\mathrm{e}^{[20]}$.

\section{AMOUNT OF COFFEE CONSUMPTION AND PROTECTIVE EFFECT ON HEPATOCARCINOGENESIS}

Several studies report not only the protective effect of coffee use on the pathogenesis of HCC but also that the protection is related to the amount of coffee ingested. Inoue et al. ${ }^{[21]}$ conducted a prospective cohort of 116,686 Japanese individuals to assess the influence of coffee consumption on the risk of hepatocellular carcinoma. Coffee proved to be a liver protective factor, with $51 \%$ lower risk of developing cancer by daily consumers compared to those who never drank coffee. An inverse relationship was also noted between consumption and incidence of this neoplasia, one or two cups per day $(\mathrm{HR}=0.52)$, three or four cups per day $(H R=0.48)$, and more than five cups per day $(H R=0.24)$.

The study by Johnson et al. ${ }^{[22]}$ analyzed 63,257 over-middle-aged Chinese individuals over approximately 5 years between 1993 and 1998. They that $18.5 \%$ of individuals did not drink coffee, $11.1 \%$ were light drinkers and drank less than one cup per day, $36 \%$ were average consumers who drank between one and two cups a day, and $34.4 \%$ were heavy drinkers and drank more than two cups of coffee a day. Of the 362 patients diagnosed with HCC, an inverse relationship was found between coffee consumption and HCC risk, with a lower risk for each increase in the number of coffee cups drunk $(P=0.05)$. Heavy consumers had a $44 \%$ lower risk of HCC than those who did not drink coffee after adjusting for potential confounders. 
A European study by Bamia et al. ${ }^{[23]}$ evaluated more than 521,000 individuals between 1992 and 2000 in 10 European countries to look for the etiologic factors of cancers and other chronic diseases. Dietary data, including coffee intake, were obtained through previously validated questionnaires. After 11 years of followup, 133 men and 68 women were diagnosed with HCC, and the results showed an average consumption of $354 \mathrm{~mL}$ per day of coffee among men and $290 \mathrm{~mL}$ of coffee per day among women. Seven percent of participants did not drink coffee. There was an inverse association between coffee consumption and the risk of HCC, and coffee consumption proved to be a liver protective factor in all groups. The higher the coffee intake, the lower the risk of liver carcinogenesis, with statistically significant results.

Aleksandrova et al. ${ }^{[24]}$ analyzed the data obtained from the European Prospective Investigation into Cancer and Nutrition, a study conducted from 1992 to 2000 on more than 520,000 individuals that aimed to establish a relationship between protection from coffee consumption and the risk of HCC in addition to serum markers of inflammation. Coffee consumption showed an inverse association with the development of HCC, with $20 \%$ more coffee consumers having an approximately $70 \%$ lower chance of HCC. Conjugate analysis of serum markers and coffee consumption showed a statistically significant reduction in all markers of liver inflammation (IL-6, glutamate dehydrogenase, alanine transaminase, aspartate transaminase, GGT, alkaline phosphatase, total bilirubin, and alpha-fetoprotein). All of these markers were directly associated with a higher risk of hepatocellular carcinoma.

\section{IMPACT OF COFFEE CONSUMPTION ON HCC MORTALITY}

Kurozawa et al. ${ }^{[25]}$, through a prospective cohort study from 1988 to 1999, evaluated the impact of coffee consumption on HCC mortality in a total of 110,792 individuals. In HCC mortality analyses, consumers of less than one cup of coffee per day had a lower risk of death from HCC, and this risk was even lower in consumers of at least one cup of coffee per day, showing a quantitative association between consumption and mortality from hepatocellular carcinoma. In subjects reporting a history of liver disease, coffee consumption was an even more prominent protective factor when compared to those who did not drink coffee or drinkers with no history of liver disease.

\section{COFFEE CONSUMPTION AND RECURRENCE OF HCC AFTER LIVER TRANSPLANTATION}

Orthotropic liver transplantation (OLT) is the therapeutic option with the most favorable outcome as it offers radical removal of the tumor and eliminates underlying chronic liver disease in selected patients or those with early-stage HCC. However, recurrence of HCC after OLT remains a serious problem with up to $20 \%$ risk. Recent studies have identified several risk factors for HCC recurrence, such as biological and radiological progression on the waiting list, number of tumor nodules, and poor differentiation ${ }^{[26]}$. However, the mechanisms are still poorly understood, and potential post-OLT strategies to prevent HCC recurrence are still needed.

Recent studies show that caffeinated coffee consumption is associated with a reduced risk of HCC recurrence and longer survival following OLT. Experimental data suggest that such benefits of coffee are associated, at least in part, with the caffeine antagonist activity in promoting adenosine receptor-mediated growth by HCC cell effects ${ }^{[27]}$.

\section{CONCLUSION}

The present review suggests that a daily habit of drinking coffee seems to protect against the development of HCC. The mechanisms may induce recurrent cycles of cell damage, repair, and regeneration in hepatocytes, leading to the formation of dysplastic nodular lesions, which are precursor lesions of HCC. Genetic and epigenetic changes may also influence HCC development. Caffeine is only one of the components of coffee; however, it appears to play a central role in protecting against the development of chronic liver disease and 
HCC. Some studies have also suggested an impact of coffee consumption on HCC mortality and recent studies showed that coffee consumption is associated with a reduced risk of HCC recurrence and longer survival following liver transplantation. These results are encouraging, mainly due to the increase in the incidence of HCC and its prognosis. Randomized studies are expected to confirm the results of mostly observational studies.

\section{DECLARATIONS}

\section{Authors' contributions}

Concept and design: Cotrim HP

Data acquisition: Carvalho KSD

Data analysis: Carvalho KSD, Cotrim HP

Manuscript preparation: Carvalho KSD, Cotrim HP

Critical revision and finalizing of the manuscript: Cotrim HP

\section{Availability of data and materials}

Not applicable.

\section{Financial support and sponsorship}

None.

\section{Conflicts of interest}

All authors declared that there are no conflicts of interest.

\section{Ethical approval and consent to participate}

Not applicable.

\section{Consent for publication}

Not applicable.

\section{Copyright}

(c) The Author(s) 2020.

\section{REFERENCES}

1. Bosetti C, Bertuccio P, Malvezzi M, Levi F, Chatenoud L, et al. Cancer mortality in Europe, 2005-2009, and an overview of trends since 1980. Ann Oncol 2013;24:2657-71.

2. Chuang SC, La Vecchia C, Boffetta P. Liver cancer: descriptive epidemiology and risk factors other than HBV and HCV infection. Cancer Lett 2009;286:9-14.

3. Trichopoulos D, Bamia C, Lagiou P, Fedirko V, Trepo E, et al. Hepatocellular carcinoma risk factors and disease burden in a European cohort: a nested casecontrol study. J Natl Cancer Inst 2011;103:1686-95.

4. Barros RK, Cotrim HP, Daltro C, Alves E, de Freitas LRA, et al. Nonalcoholic steatohepatitis in morbid obese patients: coffee consumption vs. disease severity. Ann Hepatol 2016;15:350-5.

5. Wierzejska R. Coffee consumption vs. cancer risk - a review of scientific data. Rocz Panstw Zakl Hig 2015;66:293-8.

6. Yu X, Bao Z, Zou J, Dong J. Coffee consumption and risk of cancers: a meta-analysis of cohort studies. BMC Cancer 2011;11:96.

7. Ruhl CE, Everhart JE. Coffee and caffeine consumption reduce the risk of elevated serum alanine aminotransferase activity in the United States. Gastroenterology 2005;128:24-32.

8. Bohn SK, Blomhoff R, Paur I. Coffee and cancer risk, epidemiological evidence, and molecular mechanisms. Mol Nutr Food Res 2014;58:915-30.

9. Hösel M, Quasdorff M, Wiegmann K, Webb D, Zedler U, et al. Not interferon, but interleukin-6 controls early gene expression in hepatitis B virus infection. Hepatology 2009;50:1773-82.

10. Takaki A. Control of oxidative stress in hepatocellular carcinoma: helpful or harmful? World J Hepatol 2015;7:968.

11. Murphy MP. How mitochondria produce reactive oxygen species. Biochem J 2008;417:1-13.

12. Degli Esposti D, Hamelin J, Bosselut N, Saffroy R, Sebagh M, et al. Mitochondrial roles and cytoprotection in chronic liver injury. Biochem Res Int 2012;2012:387626. 
13. Pedro JMB, Kroemer G, Filigheddu N, Galluzzi L, Porporato PE. Mitochondrial metabolism and cancer. Cell Res 2017;28:265-80.

14. Dhanasekaran R, Bandoh S, Roberts LR. Molecular pathogenesis of hepatocellular carcinoma and impact of therapeutic advances. F1000Res 2016;5:879.

15. Okano J, Nagahara T, Matsumoto K, Murawaki Y. Caffeine inhibits the proliferation of liver cancer cells and activates the MEK/ERK/ EGFR signalling pathway. Basic Clin Pharmacol Toxicol 2008;102:543-51.

16. Cavin C, Holzhaeuser D, Scharf G, Huber WW, Schilter B. Cafestol and kahweol, two coffee specific diterpenes with anticarcinogenic activity. Food Chem Toxicol 2002;40:1155-63.

17. Huber WW, Scharf G, Rossmanith W, Prustomersky S, Grasl-Kraupp B, et al. The coffee components kahweol and cafestol induce gamma-glutamylcysteine synthetase, the rate limiting enzyme of chemoprotective glutathione synthesis, in several organs of the rat. Arch Toxicol 2002;75:685-94.

18. Tanaka K, Hara M, Sakamoto T, Higaki Y, Mizuta T, et al. Inverse association between coffee drinking and the risk of hepatocellular carcinoma: a case-control study in Japan. Cancer Sci 2007;98:214-8.

19. Hu G, Tuomilehto J, Pukkala E, Hakulinen T, Antikainen R, et al. Joint effects of coffee consumption and serum gammaglutamyltransferase on the risk of liver cancer. Hepatology 2008;48:129-36.

20. Cano-Marquina A, Tarín JJ, Cano A. The impact of coffee on health. Maturitas 2013;75:7-21.

21. Inoue M, Yoshimi I, Sobue T, Tsugane S; JPHC Study Group. Influence of coffee drinking on subsequent risk of hepatocellular carcinoma: a prospective study in Japan. J Natl Cancer Inst 2005;97:293-300.

22. Johnson S, Wang R. Coffee consumption and reduced risk of hepatocellular carcinoma: findings from the Singapore Chinese Health Study. Cancer Causes Control 2012;22:503-10.

23. Bamia C, Lagiou P, Jenab M, Trichopoulou A, Fedirko V, et al. Coffee, tea and decaffeinated coffee in relation to hepatocellular carcinoma in a European population: multicentre, prospective cohort study. Int J Cancer 2015;136:1899-908.

24. Aleksandrova K, Bamia C, Drogan D, Lagiou P, Trichopoulou A, et al. The association of coffee intake with liver cancer risk is mediated by biomarkers of inflammation and hepatocellular injury: data from the European Prospective Investigation into Cancer and Nutrition. Am J Clin Nutr 2015;102:1498-508.

25. Kurozawa Y, Ogimoto I, Shibata A, Nose T, Yoshimura T, et al. Coffee and risk of death from hepatocellular carcinoma in a large cohort study in Japan. Br J Cancer 2005;93:607-10.

26. Kondili LA, Lala A, Gunson B, Hubscher S, Olliff S, et al. Primary hepatocellular cancer in the explanted liver: outcome of transplantation and risk factors for HCC recurrence. Eur J Surg Oncol 2007;33:868-73.

27. Eini H, Frishman V, Yulzari R, Kachko L, Lewis EC, et al. Caffeine promotes anti-tumor immune response during tumor initiation: involvement of the adenosine A2A receptor. Biochem Pharmacol 2015;98:110-8. 